\title{
Aortic dilatation in complex congenital heart disease
}

\author{
Koichiro Niwa \\ Department of Cardiology, Cardiovascular Center, St Luke's International Hospital, Tokyo, Japan \\ Correspondence to: Koichiro Niwa, MD, PhD, FACC, FAHA, FJCC. Department of Cardiology, Cardiovascular Center, St Luke's International \\ Hospital, 9-1 Akashi-cho, Chuo-ku, Tokyo 104-8560, Japan. Email: kniwa@aol.com; koniwa@luke.ac.jp.
}

\begin{abstract}
A bicuspid aortic valve and/or coarctation of the aorta (COA) are often associated with dilatation of the ascending aorta and para-coarctation. Congenital heart diseases (CHD), such as truncus arteriosus, transposition of the great arteries (TGA), tetralogy of Fallot (TOF), hypoplastic left heart syndrome (HLHS), single ventricle with pulmonary stenosis/atresia and the Fontan procedure, are also associated with aortic root dilatation, aneurysm and rarely, dissection, which can be fatal and require aortic valve and root surgery. A significant subset of adults with complex CHD exhibit progressive dilatation of the aortic root even after repair due to aortic medial degeneration. Medial degeneration in the ascending aorta is prevalent among the above CHD. In Marfan syndrome, bicuspid aortic valve and COA, medial degeneration is more extensive and severe than in another complex CHD. Accordingly, the incidence of ascending aortic dilatation, dissection and rupture is higher in the former. This aortic medial degeneration in CHD may either be intrinsic or secondary to the increased volume overload of the aortic root due to right-to-left shunting, or a combination of both. The association of aortic pathophysiological abnormalities, aortic dilatation and aorto-ventricular interaction is clinically referred to as "aortopathy". The major purpose of medical treatment for aortopathy is to reduce the structural changes within the aortic wall and slow down the progression of aortic dilatation to reduce the risk of cardiovascular events. Several medications have been studied, including -blockers, angiotensin II type I (AT1) receptor blockers (ARBs) and Ca-antagonists. However, the results, except for Marfan syndrome, are limited. In aortopathy, concomitant aortic valve and aortic root repair/replacement is required. The Bentall operation comprises root replacement with an aortic tube graft and mechanical valve, and has been widely applied. On the other hand, valve-sparing operations have been developed in order to preserve the aortic valve.
\end{abstract}

Keywords: Aortopathy; aortic medial degeneration; aortic dilatation; aortic aneurysm; aortic dissection

Submitted Jul 30, 2018. Accepted for publication Dec 17, 2018.

doi: $10.21037 /$ cdt.2018.12.05

View this article at: http://dx.doi.org/10.21037/cdt.2018.12.05

\section{Introduction}

Dilation of the ascending aorta in congenital heart diseases (CHD) may exceed hemodynamic or morphogenetic expectations, or the aorta may become aneurysmal and rupture $(1,2)$. Aortic dilation in Marfan syndrome, bicuspid aortic valve and coarctation of the aorta $(\mathrm{COA})$ is well documented, and these disorders are associated with ascending aortic and/or para-coarctation medial degeneration $(3,4)$. CHD, such as single ventricle with pulmonary stenosis/atresia, truncus arteriosus communis (TAC), complete transposition of the great arteries (TGA), hypoplastic left heart syndrome (HLHS), tetralogy of Fallot (TOF) and the Fontan procedure, are also associated with aortic medial degeneration and aortic dilatation (1). These observations led us to hypothesize that many CHD cause aortic medial degeneration that reflects a common developmental fault, which weakens the aortic wall.

There are reported variables that alter the structure of ascending aortic media in humans such as aging, hypertension, pregnancy (via increased hemodynamics in the setting of genetic abnormality), chromosomal abnormality and genetics (2) (Table 1). This aortic dilatation in CHD may either be intrinsic or a secondary result from 
Table 1 Variables that affect the structure of the ascending aortic media

Variables
Systemic hypertension
Aging
Pregnancy
Chromosome abnormality: Marfan syndrome, Turner syndrome
and Noonan syndrome
Gene abnormality: fibrillin-1 defect (15q21.1)
Deletion of the TGF- $\beta 1$ receptor and ALK5 signaling in neural
crest cells
Metalloproteinase and elastin
Hemodynamic abnormality (increased aortic blood flow);
complex CHD with PS/PA)
Intrinsic abnormality of the aortic wall in congenital heart
diseases
Chromosome $22 q 11.2$ deletion

TGF- $\beta$, transforming growth factor- $\beta$; PS, pulmonary stenosis; PA, pulmonary atresia.

Table 2 Complex CHD associated with aortic dilatation in adults

TOF

Single ventricle with pulmonary atresia or stenosis

TAC

Transposition of the great arteries

Hypoplastic left heart syndrome

Fontan procedure

CHD, congenital heart diseases; TOF, tetralogy of Fallot; TAC, truncus arteriosus communis.

increased volume overload of the aortic root (right to left shunting), or a combination of both. In this review, we will discuss the aortic complications of several complex CHDs, with emphasis on their pathophysiology, clinical manifestations and outcomes. Moreover, medications and interventions for aortopathy in complex CHD are described.

\section{CHD associated with aortic dilatation}

Many CHD associated with aortic dilatation are shown in Table 2.

\section{Histopathological abnormalities and cause of aortic dilatation in several complex CHD}

A significant subset of adults with CHD exhibits progressive dilatation of the aortic root even after repair due to medial abnormalities that may lead to aortic regurgitation (AR) and predispose them to dissection and rupture, which can be fatal and require aortic valve and aortic root surgery. Aortic medial degeneration is most severe in Marfan syndrome, and is prevalent and qualitatively similar in a variety of CHD with a wide age range $(1,5)$. Medial degeneration is incorrectly called "cystic medial necrosis". Medial degeneration is defined as disorganization, thinning and fragmentation of elastic fibers, a conspicuous increase in collagen, mucoid extracellular matrix accumulation, and smooth muscle cell disorganization and nuclei loss. Necrotic and/or cystic lesions are not found in medial degeneration. In CHD, aortic medical degeneration is less extensive and less severe than in Marfan syndrome (1). Accordingly, the incidence of ascending aortic dilatation, dissection and rupture is lower, and the aortic root medial lesions are less severe in CHD patients with aortic dilatation (1). This aortic medial degeneration in CHD is either caused by intrinsic factors or volume overload of the aorta due to primary right-to-left shunting (6). Niwa et al. (1) reported that in 88 (10 different diagnoses) CHD patients with dilated aorta aged 3 weeks to 81 years ( $32 \pm 6$ years) ( 48 males, 40 females), surgical biopsy of aortic specimens revealed some degree of medial degeneration in all patients (Figure 1). In their study, surgical biopsies and necropsy specimens from the ascending aorta (or in COA, the para-coarctation site: above and below the coarctation) were obtained at the time of surgery. Specimens for light microscopy (LM) were fixed in formaldehyde, and then used for hematoxylin-eosin, elastica van Gieson, trichrome and colloidal iron staining. Tissue for transmission electron microscopy (EM) was immediately fixed by immersing in $2.5 \%$ glutaraldehyde and $2 \%$ paraformaldehyde, and then post-fixed with $2 \%$ osmium tetroxide, stained with uranyl acetate and lead citrate, and embedded in an epoxy resin. Semi-thin sections were stained with toluidine blue for screening, selection and an overview for semi quantitative grading. Ultra-thin sections were examined with a Zeiss EM 109 electron microscope. Medial degeneration was defined as the existence of disruption and fragmentation of elastic fibers, a conspicuous increase in collagen and loss of smooth muscle cells (Figure 2). Medial degeneration on LM and EM was classified semi- quantitatively (according 


\begin{tabular}{|c|c|c|c|c|}
\hline & Normal & Grade 1 & Grade 2 & Grade 3 \\
\hline Marfan & & & & 10 \\
\hline AAE & & & & 5 \\
\hline BAV AS & & 2 & 6 & 4 \\
\hline BAV AR & & 4 & 3 & 3 \\
\hline TOF & & & 9 & 6 \\
\hline SV PS & & & 2 & 1 \\
\hline TA PS & & & 2 & 1 \\
\hline DORV & & & 1 & 1 \\
\hline DOLV & & & 1 & \\
\hline VSD & & & & 1 \\
\hline Do.Ao A & & & 1 & \\
\hline PTA & & & 3 & 2 \\
\hline d-TGA & & & 6 & 2 \\
\hline Controls & 21 & & & \\
\hline
\end{tabular}

Figure 1 Histological severity of the aortic media according to aortic biopsy specimens in CHD patients with a dilated aorta (n=88) by light and electron microscopy. Medial degeneration on light and electron microscopy was classified as: absent; non-specific (Grade 1), abnormal; moderate (Grade 2) or abnormal; severe (Grade 3). Specimens were stained by hematoxylin-eosin, elastica van Gieson, trichrome \& colloidal iron. AAE, annulo-aortic ectasia; BAV AS, bicuspid aortic valve with aortic stenosis; AR, aortic regurgitation; TOF, tetralogy of Fallot; SV PS, single ventricle with pulmonary stenosis; TA, tricuspid atresia; DORV, double outlet right ventricle; DOLV, double outlet left ventricle; VSD, ventricular septal defect; Do Ao A, double aortic arch; PTA, persistent truncus arteriosus; d-TGA, d-transposition of the great arteries; Controls, transplant donor heart.

to severity and extent): absent; non-specific (Grade 1), abnormal; moderate (Grade 2), or abnormal; severe (Grade 3). Widespread disruption and fragmentation of elastic fibers, a conspicuous increase in collagen and widespread loss of smooth muscle cells, Grade 3 (positive controls) - large areas of the complete loss of elastic fibers, a conspicuous increase in collagen and ground substances, and overall loss of smooth muscle cells. As shown in Figure 1, Grade 3 lesions were found as follows: 10/10 in Marfan syndrome and 5/5 in annuloaortic ectasia. Grade $2-3$ lesions were found as follows: $15 / 15$ in TOF, $3 / 3$ in single ventricle (Fontan procedure), $3 / 3$ in tricuspid atresia (Fontan procedure), TGA in $8 / 8$, double outlet right ventricle (Fontan procedure) in $2 / 2$, and truncus arteriosus in $5 / 5$.

\section{Multiple CHD and aortopathy}

TOF

Clinical findings/definitions, pathophysiology and late complications

TOF is a monology of right ventricular outflow tract (RVOT) dysplasia, but there are 4 classic defects: pulmonary stenosis (RVOT stenosis), malalignment type ventricular septal defect (VSD), right ventricular hypertrophy and an overriding of the aorta. The surgical procedure for TOF involves increasing the size of the pulmonary valve and pulmonary arteries with patching and RVOT with resection of RVOT muscles, and closing the VSD. In babies with a pulmonary artery that is too small, temporary palliative surgery (usually Blalock-Taussig 

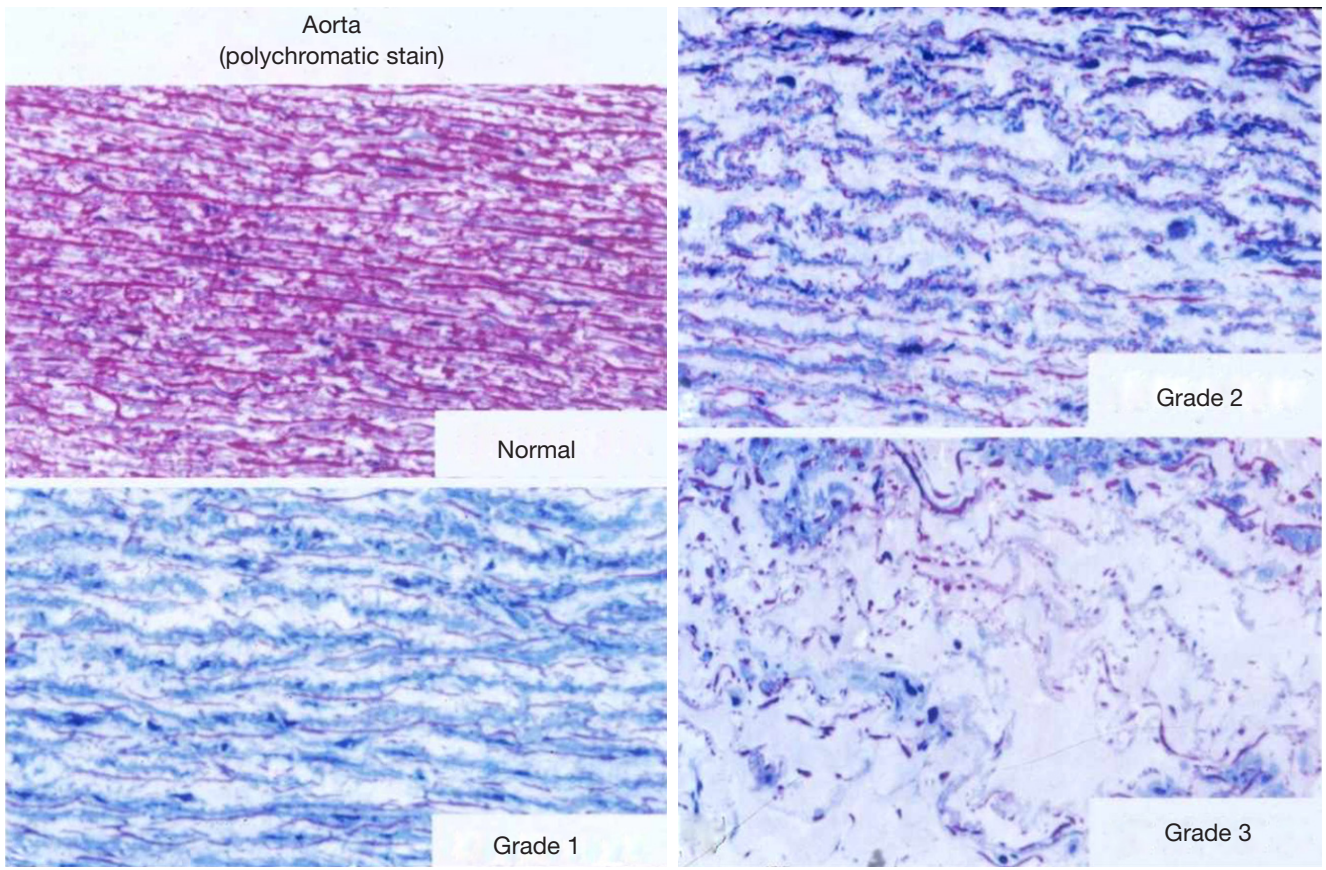

Figure 2 Light microscopic semiquantitative grading system (polychromatic staining). Normal (negative controls): closely packed long parallel arrays of intact elastic fibers; Grade 1: mild fragmentation of elastic fibers, a mild increase in collagen; Grade 2: widespread disruption and fragmentation of elastic fibers, a conspicuous increase in collagen; widespread loss of smooth muscle cells; Grade 3 (positive controls): large areas of the complete loss of elastic fibers, a conspicuous increase in collagen and ground substance and overall loss of smooth muscle cells.

shunting) may be performed with plans for a future second reparative surgery. Late complications in TOF after repair are as follows: (I) right ventricular dysfunction due to residual pulmonary stenosis or residual VSD, or longlasting pulmonary regurgitation; (II) arrhythmias (sick sinus syndrome, complete atrioventricular block, atrial flutter fibrillation, paroxysmal supraventricular tachycardia and ventricular tachycardia); (III) aortic root dilatation, AR; (IV) left ventricular dysfunction due to ventricular interaction.

Then, reoperation (pulmonary valve replacement, RVOT reconstruction, VSD closure, aortic valve replacement aortic repair) is performed in some cases in adulthood.

\section{Pathophysiology and cause of aortic root dilatation in TOF}

Aortic dilatation in TOF may either be intrinsic or a secondary result from increased volume overload of the aortic root (right-to-left shunting) or genetic, or a combination of these. John AS found aortic dilation in 10 out of 93 (10.8\%) patients with chromosome 22q11.2 partial deletion without conotruncal abnormality (7). In a series of TOF patients, chromosome 22q11.2 partial deletion was suggested to be a risk factor of aortic dilatation (8).

\section{Unrepaired TOF and aortic dilatation}

Aortic dilatation is a well-known feature of unrepaired TOF and is well correlated with the severity of RVOT stenosis. Moreover, it is greatest in TOF and pulmonary atresia. The size of the ascending aorta is large in fetuses with TOF on fetal echocardiography, and this aortic dilatation is due to medial degeneration coupled with prior longstanding volume overload of the ascending aorta (right-to-left shunting through malalignment-type VSD) (9) (Figure 3). In a previous study (10), biopsied sections were examined under light microscopy for histological abnormalities. They found that Grade 2 or 3 medial degeneration was present in the ascending aorta in $59 \%$ of TOF patients, including infants. This suggests that medial degeneration exists before intra-cardiac repair. Among cyanotic CHD, TOF was the first in which aortic dilation was noted $(11,12)$.

\section{Repaired TOF and aortic dilatation}

A significant subset of adults exhibits progressive aortic root dilatation late after repair of TOF, which may lead to AR, 

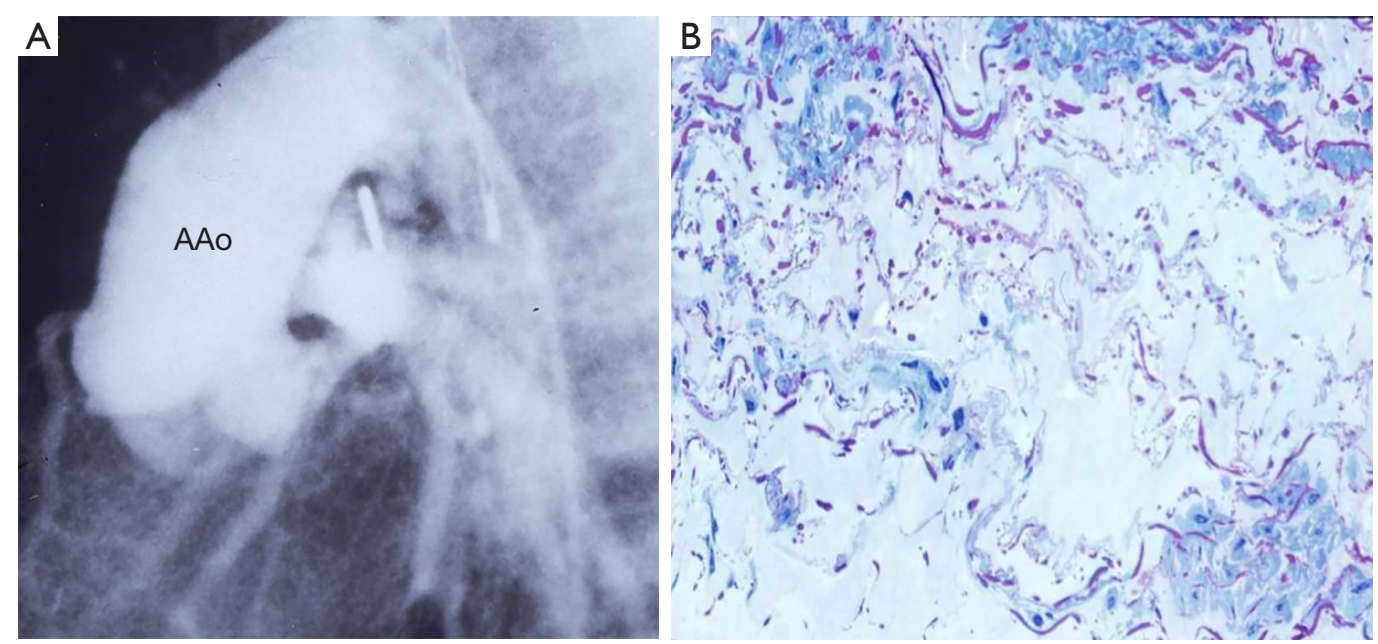

Figure 3 Dilated ascending aorta (AAo) in a 3-year-old boy with a VSD and pulmonary atresia. (A) Dilated AAo with hypoplastic pulmonary artery (aortic angiography); (B) Grade 2 abnormality in the aortic media (light microscopy, elastica van Gieson staining).

and predispose them to dissection and rupture (9). Thus, the aortic root can dilate even after elimination of rightto-left shunting. It was reported that $15 \%$ of adults with repaired TOF had a dilated aortic root $>40 \mathrm{~mm}$ (8). Risk factors for aortic dilatation after TOF repair are related to specific hemodynamic abnormalities, such as pulmonary atresia, right aortic arch and a history of aorto-pulmonary shunting, and patient demographics such as male sex and chromosome 22q11 deletion (8). Chong et al. (13) examined 67 children after TOF repair, and noted aortic dilation (Z-score $>2$ ) in $88 \%, 87 \%, 61 \%$ and $63 \%$ at the annulus, sinus of Valsalva, sinotubular junction and ascending aorta, respectively. Currently, at least 5 case reports on repaired TOF and aortic dissection have been published (14-19). All of these patients had an aortic root size equal to or greater than $55 \mathrm{~mm}$ (9) (Table 3). Different from Marfan syndrome, aortic aneurysm and dissection/rupture have been rarely reported in TOF. This may be because medial degeneration in the aorta in TOF is of a lower grade and distribution than that in Marfan syndrome (1).

\section{Prevention and management of progressive aortic dilatation in TOF}

There is no current consensus on $\beta$-blocker or angiotensin II receptor blocker (ARB) administration to limit progressive dilatation of the aortic root in patients with repaired TOF. There are several papers on ascending aortic repair $(19,26)$. Dodds et al. (26) reported aortic root rerepair in 6 patients with TOF/VSDs with pulmonary atresia who were followed-up for 13.5 years after initial repair.
They performed lateral aneurysmorrhaphy in 5 and graft replacement of the ascending aorta in one. All patients are doing well after repair. Mizuno et al. (27) reported that among 236 of 4,010 repaired TOF patients (current age $>15$ years), 7 ( $3 \%$ ) required reoperation due to aortic dilatation.

\section{Key points}

* One late complication after repaired TOF is aortic root dilatation;

- Progressive aortic root dilatation is exhibited late after repair of TOF;

- Aortic dilatation in TOF may be intrinsic (medial degeneration) or a secondary result of the increased volume overload of the aortic root (right-to-left shunting), genetic or a combination of both.

\section{HLHS}

Clinical findings/definitions, pathophysiology and late complications

HLHS is a rare CHD in which the left side of the heart is markedly underdeveloped. It may affect the left ventricle, aorta, aortic valve or mitral valve, including aortic atresia/ severe stenosis and mitral atresia/severe stenosis. Currently, infants with HLHS undergo staged reconstructive surgery (Norwood or Sano procedure), followed by the Glenn or Hemi Fontan procedure, and then the Fontan procedure is completed. In the Norwood or Sano procedure, the right ventricle is used to pump blood into the systemic 
Table 3 Case reports of ascending aortic dissection $(n=10)$ and large aneurysm $(n=1)$

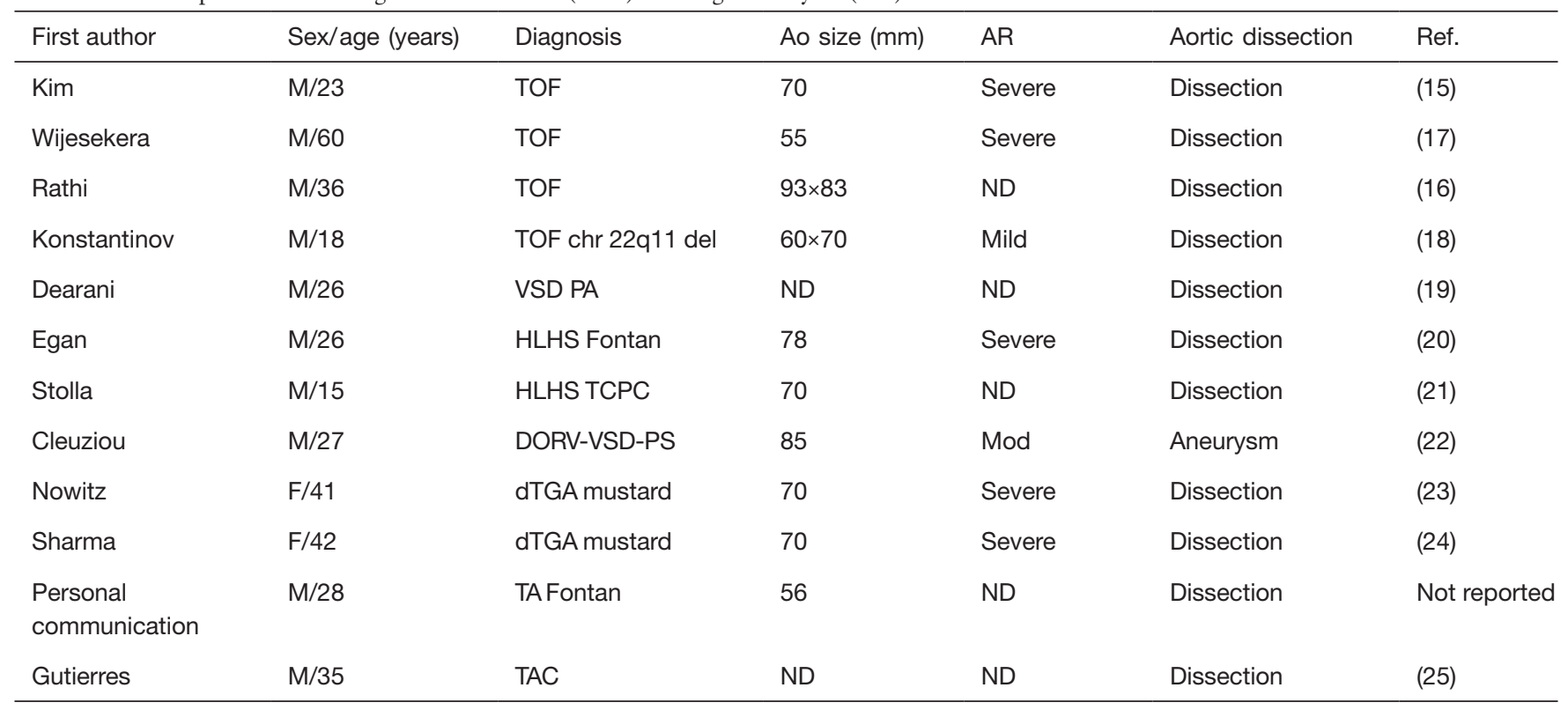

M, male; F, female; TOF, tetralogy of Fallot; VSD PA, ventricular septal defect pulmonary atresia; ND, not described; HLHS, hypoplastic left heart syndrome; TCPC, total cavopulmonary connection; DORV, double outlet right ventricle; PS, pulmonary stenosis; TGA, transposition of the great arteries; TA, tricuspid valve atresia; TAC, truncus arteriosus communis.

circulation. Using a shunt, deoxygenated blood is passed through the pulmonary artery. Either the subclavian artery can be connected to the pulmonary circulation (BlalockTaussig shunting) or a shunt can be made directly from the right ventricle to the pulmonary circulation (Sano shunt). The narrow aorta is enlarged using a patch to improve blood flow to the body. Then, the final surgery is the Fontan procedure. Late complications are the same as those after the Fontan procedure, which are described in the section on the Fontan procedure.

\section{Pathophysiology and cause of aortic root dilatation in HLHS}

Although the pathophysiology of aortic root dilation in patients with HLHS is largely unknown, the aortic dilatation is considered to be related to anatomical, genetic and hemodynamic factors. Reconstructive surgery of the aorta (the Norwood or Sano procedure) may be one of the causes of future progressive aortic dilatation. Several studies have suggested that HLHS is heritable and genetically related to bicuspid aortic valve, which is known to be associated with aortopathy $(27,29)$. The recurrence risk of bicuspid aortic valve in the families of patients with HLHS is similar to that for isolated bicuspid aortic valve patients. Linkage to multiple chromosomal regions, such as $10 \mathrm{q} 22$ and $6 \mathrm{q} 23$, was identified in the kin of HLHS patients, suggesting the genetic heterogeneity of HLHS (28). Moreover, a chromosomal locus on $14 \mathrm{q} 23$ was identified for both HLHS and bicuspid aortic valve, which provided the first direct evidence of a genetic similarity between these 2 disorders (29). Further elucidation of risk factors for progressive aortic dilation in HLHS as well as its pathological mechanisms is required.

\section{Aorta in unrepaired TGA}

There is no evidence of aortic root dilatation in unrepaired TGA.

\section{Aortic root dilatation in HLHS before the Norwood or Sano procedure}

During the fetal stage and after birth before initial surgery, the aortic root is hypoplastic. Therefore, no aortic dilatation exists before the Norwood or Sano procedure.

\section{Aortic root dilatation in repaired HLHS (post-Fontan procedure)}

Patients with HLHS who undergo the Fontan procedure can now survive to adulthood, but aortopathy is one of the major complications. Neo-aortic root dilation after staged reconstruction for HLHS is a well-known complication of 
HLHS, and it often progress over time. Cohen et al. (30) followed 53 patients with HLHS after the Fontan procedure for 9 years and found that the neo-aortic root progressively dilated out of proportion to the body size, with $98 \%$ having a Z-score $>2$ and neo-AR developed in $61 \%$. Furthermore, there is growing evidence of aortic dilatation in patients with HLHS after palliative surgery and/or Fontan repair (31-33). Post-Fontan procedure patients with HLHS have a dilated descending aorta (30), different from other postFontan procedure patients who have a small descending aorta.

\section{Prevention and management of progressive aortic dilatation in HLHS}

No specific recommendations for the management of aortic dilation in HLHS are currently available.

\section{Key points}

* Progressive neo-aortic root dilation after staged reconstruction for HLHS is a well- known complication of HLHS;

* Aortic dilatation in HLHS is considered to be related to anatomical, genetic and hemodynamic factors.

\section{Complete transposition of the great artery (TGA) after the arterial switch operation (Fatene procedure) or atrial switch operation (Mustard, Senning procedure)}

Clinical findings/definitions, pathophysiology and late complications

In TGA, the positions of the pulmonary artery and the aorta are switched. Two major types of surgery can correct TGA. The first procedure creates a tunnel (a baffle) between the atria. This redirects the pulmonary venous blood to the right ventricle and aorta, and the infra/supra vena caval blood to the left ventricle and the pulmonary artery. This operation is called the Mustard procedure or the Senning procedure. The most important complications in adults after repair are systemic right ventricular failure, systemic $\mathrm{AV}$-valve (tricuspid valve) regurgitation supraventricular arrhythmias and baffle stenosis.

The second type of procedure is the arterial switch operation. The aorta and pulmonary artery are switched back to their normal positions. The aorta is connected to the left ventricle and the pulmonary artery is connected to the right ventricle. The coronary arteries also are reattached to the new aorta (originally the pulmonary artery). Late complications in adults after repair are coronary artery stenosis, pulmonary stenosis, aortic regurgitation and aortic dilatation.

\section{Pathophysiology and cause of aortic root dilatation in TGA}

Lalezari et al. conducted histological examinations of the pulmonary artery and aorta in neonates and infants with unrepaired TGA (34). Routine histology and immunohistochemical staining for smooth muscle cell differentiation markers, alpha-smooth muscle actin, SM22 and calponin, were performed. In the early stage of TGA, no marked differences were found between the aorta and pulmonary artery. With increasing age, however, there was marked down-regulation of all smooth muscle cell markers in the pulmonary artery. They concluded that this structural abnormality may explain the neoaortic root dilatation that has been reported as a late complication of the arterial switch operation. In addition, aortic medial degeneration is observed in neonates with TGA after the arterial switch operation in both the neo-aorta and pulmonary artery (35).

\section{Unrepaired TGA}

There is no evidence of aortic dilatation in TGA before intracardiac repair $(34,35)$.

The aorta in TGA after the arterial switch operation The aorta in TGA after the arterial switch operation has unique anatomical and operative features. The pulmonary valve and main pulmonary artery are used to form the neoaortic valve and ascending aorta. Aortic dilation is a wellknown complication after the arterial switch operation in TGA (36,37). Progressive dilation of the neo-aortic root exceeds somatic growth during a longer follow-up period. Although there is variation among individuals, aortic dilatation generally progresses. Schwartz et al. (38) reported that in 335 children who underwent the arterial switch operation, half had evidence of neo-aortic root dilatation at 10 years after the surgery, and the 10-year rate of freedom from re- operation of the aortic root or valve was $95 \%$. Angeli et al. (36) described 803 patients who previously underwent the arterial switch procedure, and found that neo-aortic root dilatation was common and surgical intervention was performed for only 3 patients.

McMahon reported that development of severe neo-aortic root enlargement was associated with prior pulmonary artery (PA) banding, the presence of a VSD and Taussig-Bing anatomy, but was independent of coronary arterial anatomy, coronary arterial transfer technique and 
associated lesions (38-40).

\section{The aorta in TGA after the atrial switch operation}

For patients with TGA who have undergone the atrial switch operation, data regarding the incidence and degree of long-term aortic root dilation are lacking. However, a single case report of surgical repair of aneurysms of the ascending aorta and progressive AR after atrial switch operation has been described (41). Recently, Yurasek et al. (42) conducted a retrospective database review on aorta and pulmonary arterial dimensions in 39 patients, with a median age of 29 , with TGA and intact ventricular septum who underwent the atrial switch operation using cardiac magnetic resonance. Their data was then compared with 40 normal controls. Sizes of the annulus, sinotubular junction and root were measured. All these measurements in both the aortic root and pulmonary artery were significantly dilated compared with those in controls. Therefore, adult patients with TGA after the atrial switch operation have a greater incidence of dilation of both the pulmonary artery and aorta. The risk factors for aortic dilatation observed in TGA after the arterial switch operation $(38,40)$ cannot be applied to patients with TGA after the atrial switch operation.

\section{Key points}

* Progressive dilation of the neo-aortic root exceeds somatic growth after a longer follow-up period in TGA after the arterial switch operation;

* Marked neo-aortic root enlargement is associated with prior pulmonary artery banding, the presence of a VSD and history of Taussig-Bing anatomy;

- Adult patients with TGA after the atrial switch operation have a greater incidence of dilation of both the pulmonary artery and the aorta.

\section{Fontan procedure}

Clinical findings/definitions, pathophysiology and late complications

The Fontan procedure is performed for patients who possess only a single functional ventricle where a biventricular repair is impossible or inadvisable. It involves diverting the venous blood from the inferior vena cava (IVC) and superior vena cava (SVC) to the pulmonary arteries without passing through the morphologic right ventricle. The Fontan procedure includes several different surgical modifications, where atriopulmonary connection, intracardiac total cavo pulmonary connection (lateral tunnel) and extracardiac total cavo pulmonary connection are today the most important. There are many late complications, including atrial thrombus/systemic embolization, systemic ventricular dysfunction/failure, atrial flutter/fibrillation, protein losing enteropathy (PLE), increased cyanosis, enlarged right atrial size, obstructed Fontan circuit (conduit obstruction), FALD (Fontan-associated liver disease), and aortopathy (aortic dilatation) and vasculopathy (femoral vein dysfunction).

Pathophysiology and cause of aortic root dilatation in patients after the Fontan procedure

Fontan procedure candidates (cyanotic CHD with single systemic functional ventricle, and pulmonary stenosis, pulmonary atresia or post-pulmonary artery banding) usually have either pulmonary stenosis or pulmonary atresia. Sometimes they have previously undergone the Glenn procedure or Blalock-Taussig shunting. In these patients, the Qp/Qs (pulmonary blood flow/aortic blood flow ratio) is under 1.0. The aortic flow is much larger than the pulmonary artery flow. Therefore, aortic dilatation is often observed in these patients before the Fontan procedure due to the low $\mathrm{Qp} / \mathrm{Qs}$ combined with aortic medial degeneration (1).

Robust evidence of the dilated aorta in patients has been reported after the Fontan procedure (43-49). Some of these etiologies are intrinsic (medial degeneration) in nature; however, traditional risk factors, such as heart failure and metabolic factors, are now being recognized as significant additional contributors to Fontan aortopathy.

\section{The aorta before the Fontan procedure.}

In HLHS, as mentioned earlier, the aorta gradually dilates out of proportion to somatic growth. In other cyanotic CHD patients except those with HLHS who are candidates for the Fontan procedure, aortic dilatation is often observed due to a higher systemic blood volume and aortic medial degeneration (1).

\section{The aorta after the Fontan procedure}

Evidence of aortic dilatation has been reported in patients after the Fontan procedure, and most of the studies focused on the dilated and stiffened ascending aorta (43-49). Patients after the Fontan procedure have a dilated ascending aorta with a smaller descending aorta and other peripheral arteries, possibly due to low cardiac output originally from Fontan circulation (Figure 4). However, patients after the 

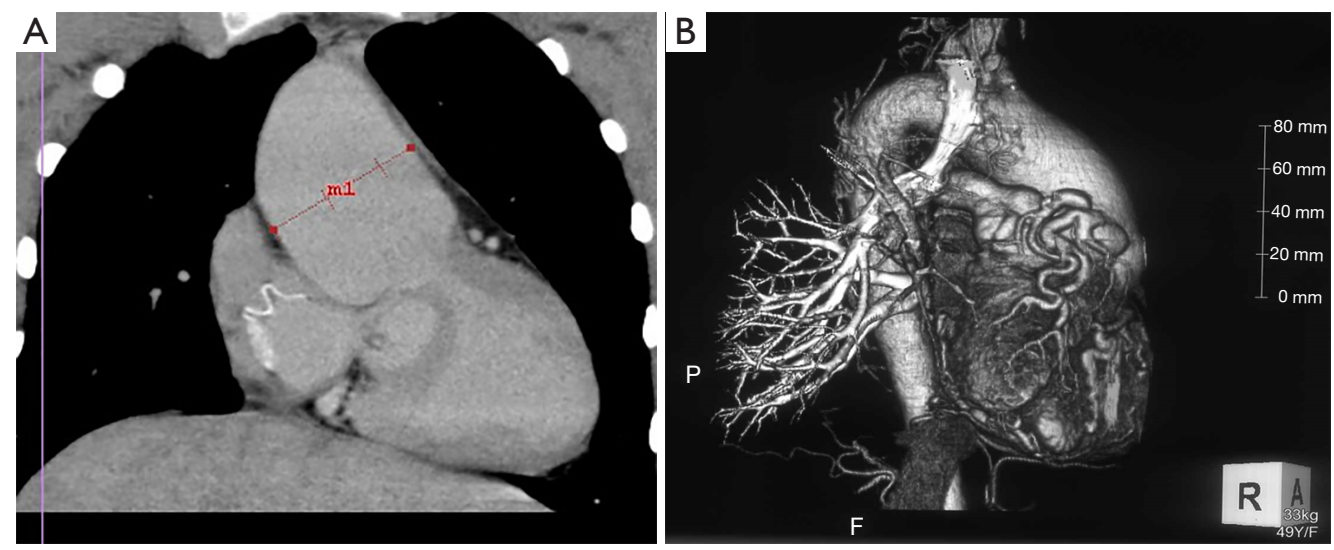

Figure 4 Dilated aorta in patients with cyanotic congenital heart disease (CT angiography). (A) Aortic aneurysm (53 mm in diameter: $\mathrm{m} 1$ ) in a 17-year-old boy with pulmonary atresia and an intact ventricular septum after the Fontan procedure, and mild aortic regurgitation; (B) dilated ascending aorta $(50 \mathrm{~mm})$ in a 50-year-old female with tricuspid atresia and right aortic arch after the Glenn procedure, and moderate aortic regurgitation.

Fontan procedure with HLHS have a dilated descending aorta (48). In a recent study on 130 consecutive patients by Ohuchi et al. (48), the prevalence of dilated ascending aorta (standardized by body surface area) equal to or greater than $1 \mathrm{SD}$ and equal to or greater than $2 \mathrm{SD}$ was $46.9 \%$ and $26.2 \%$, respectively. The standardized ascending aorta was slightly larger after the Fontan procedure with nonleft ventricular morphology of the systemic ventricle than in those with the left ventricle as the systemic ventricle $(106 \pm 18 \%$ vs. $113 \pm 24 \%)$. Age, body mass index and cardiac index were closely associated with dilatation of the ascending aorta.

Aortic dissection was reported in a patient after the Fontan procedure with HLHS and a markedly dilated aortic root (78 $\mathrm{mm}$ in diameter) (20). Another patient, 28 years old, with tricuspid atresia with an aortic root size of $56 \mathrm{~mm}$ developed aortic dissection after the Fontan procedure (Table 3).

\section{Key points}

* Approximately half of the patients have a dilated ascending aorta after the Fontan procedure;

- Age, body mass index and cardiac index are closely associated with dilatation of the ascending aorta;

- Patients after the Fontan procedure have a dilated ascending aorta with a smaller descending aorta. In contrast, patients with HLHS after the Fontan procedure have a dilated ascending aorta and descending aorta.

\section{TAC (truncus arteriosus)}

Clinical findings/definitions, pathophysiology and late complications

The embryological structure known as the truncus arteriosus fails to properly divide into the pulmonary trunk and aorta. Thus, there is a single artery arising from the two ventricles, which gives rise to both the aortic and pulmonary vessels with a large VSD. Regarding surgical repair, the pulmonary arteries are detached from the common truncal artery and connected to the right ventricle using a conduit. Late complications include pulmonary stenosis (conduit stenosis and valvular stenosis) and aortic dilatation. The conduit may need to be replaced as the child grows. Furthermore, the truncal valve is often abnormal and may require future surgery to improve its function.

\section{Pathophysiology and cause of aortic root dilatation in TAC}

One arterial trunk arises from the heart, and supplies mixed blood to the pulmonary arteries and systemic circulation. Arterial trunk is dilated due to the burden of the combined pulmonary artery and systemic blood flow.

\section{The aorta before surgical repair}

A dilated aortic root is found in the majority of truncus arteriosus patients regardless of repair (1) (Figure 5). However, there is only one report of dissection of the aorta in a 35 -year-old unrepaired man with irreversible 

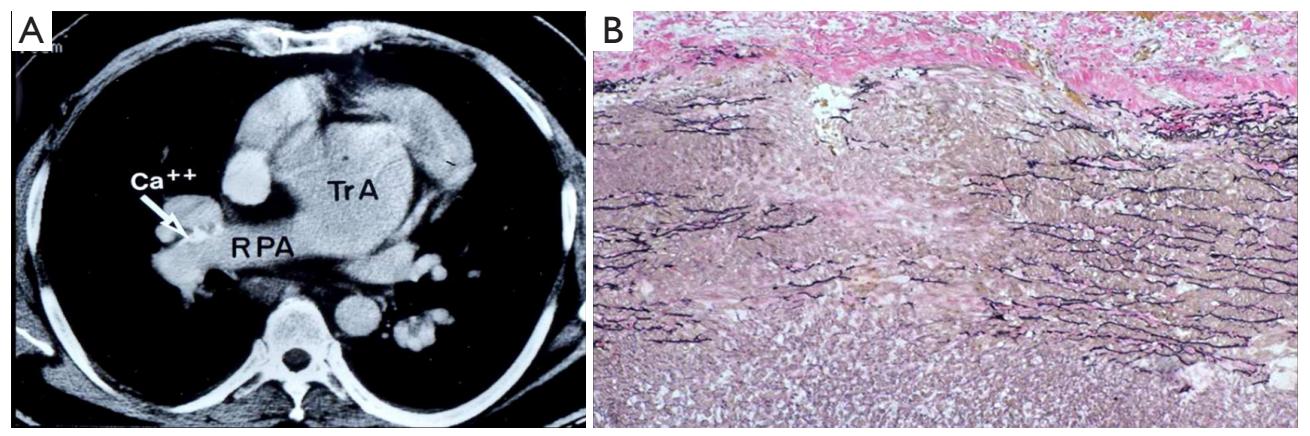

Figure 5 Dilated ascending aorta in a 43-year-old male with persistent truncus arteriosus. (A) Dilated truncal artery (Tr A) with the right pulmonary artery (RPA) directly coming out from the Tr A. Calcium deposition was observed in the RPA (CT angiography); (B) Grade 2 abnormality in the aortic media was noted (light microscopy).

pulmonary hypertension (25). He had chest pain and suddenly died due to cardiac tamponade caused by intramural-limited dissection and rupture of the common trunk.

The aorta after extracardiac conduit operation Carlo et al. (50) identified 76 truncus arteriosus patients after repair with a median age of 5.4 years (range, $0-32.7$ years), and found that the mean truncal root $Z$-score was $5.1 \pm 2.3$, and 3 had truncal root $Z$-scores greater than or equal to 2. Most patients had truncal root diameters greater than or equal to a $Z$-score of 2 , comprising $96 \%$ of their cohort. Six patients underwent truncal root surgery, typically for indications of root dilation with significant truncal valve regurgitation and left ventricular dilation. To our knowledge, there are no reports on aortic dissection in persistent truncus arteriosus patients after repair.

Truncal valve regurgitation is often associated with truncus arteriosus. Henaine et al. (51) examined 153 repaired patients with truncus arteriosus, and reported that $63 \%$ of the patients were free from truncal valve re-operation at 18 years of age.

\section{Key points}

* A dilated aortic root is found in the majority of truncus arteriosus patients regardless of repair;

- The cause of aortic dilatation is mainly due to its embryological structure, known as the truncus arteriosus (combined pulmonary trunk and aorta).

\section{Medical prophylaxis}

The major purpose of medical treatment for aortopathy is to reduce the structural changes within the aortic wall and slow down the progression of aortic dilatation to reduce the future possible risk of aortic and ventricular remodeling. $\beta$-blockers have been the mainstay of medical treatment for many years for Marfan syndrome. Recently, losartan, an angiotensin II type I (AT1) receptor blocker (ARB), has been demonstrated to be promising in a mouse model of Marfan syndrome, and subsequently in patients with Marfan syndrome (52-54). Several clinical studies focusing on the modulation of the angiotensin II pathway proposed angiotensin-converting enzyme (ACE) inhibitors as other alternatives or adjuncts to the standard treatment with $\beta$-blockers $(54,55)$. A combination therapy with losartan and $\beta$-blockers may offer more protection, especially for those with severe or progressive aortic dilatation due to Marfan syndrome. ARB or ACE inhibitors may be considered as an alternative treatment to $\beta$-blockers for Marfan syndrome patients, but there are no data on the effectiveness of these medications in adults with CHD (ACHD). Furthermore, $\beta$-blockers, particularly the 2 nd generation drugs, have negative effects on the lipid and glucose profiles, do not reduce central pulse pressure and have minor effects on TGF- $\beta$ signaling. Thus, care must be taken for ACHD patients. In addition, good control of the risk factors, such as systemic hypertension, dyslipidemia and diabetes, smoking cessation and healthy lifestyle modifications with suitable exercise are also important to delay the progression of aortic dilatation.

\section{Pregnancy and delivery}

During normal pregnancy, the total blood volume increases by $40 \%$ to $50 \%$ by the end of the second trimester. 
Moreover, peripheral resistance decreases by 16 weeks of gestation to adapt to the increased uterine blood flow, but it becomes higher than that before conception due to the increased renin and angiotensin II levels. These hemodynamic changes may cause dilatation and dissection of the aorta in the mother, especially in those with weak aortic media due to Marfan syndrome or Loeys-Dietz syndrome (56). During normal pregnancy, the estrogen level is high to support the growth of the uterus, the fetus and the mammary glands. Exposure to estrogen during pregnancy makes the elastic fibers of the aortic media fragile (aortic medial degeneration) (57). Furthermore, the renin-angiotensin system is affected, which increases not only the extracellular fluid and volume load, but also the vulnerability of the aortic wall. Pregnancy and delivery are risk factors for aortic dissection in Marfan syndrome patients $(58,59)$. There is no report on dissection in other CHD patients with a dilated aorta during pregnancy and delivery, but great care must be taken for women with CHD and dilated aortic root. $\beta$-blockers should be continued by Marfan syndrome patients during pregnancy; however, they can induce fetal growth retardation and hypoglycemia. There is no consensus regarding $\beta$-blocker administration for patients with CHD with a large aorta during pregnancy and delivery. ACE inhibitors and ARB are toxic to the fetal kidney, and cause teratogenicity of CHD and reduce the amniotic fluid volume (60). These medications should not be recommended to female patients of childbearing age with CHD.

\section{Surgery}

To avoid acute aortic dissection or rupture, prophylactic surgery on the ascending aorta should be performed when indicated according to the American Heart Association (AHA) and European Society of Cardiology (ESC) guidelines $(61,62)$. The aortic diameter is a major criterion for elective replacement of the ascending aorta. These recommendations are markedly influenced by the underlying etiology and histopathology of aortic disease, such as patients with bicuspid aortic valve or Marfan syndrome, as well as other specific risk factors. However, the indication for surgery for CHD patients with a dilated aorta has not been established. Case reports of aortic dissection in CHD are presented in Table 3. Several CHD are included, but the aortic root size in patients with CHD and aortic dissection has been reported to range from 56 to $93 \mathrm{~mm}$ (63). Stulak et al. (64) found that moderate ascending aortic enlargement is common among patients with conotruncal anomalies before aortic valve surgery. However, aortic dissection and the subsequent need for reoperation are infrequent in this setting; therefore, the moderately dilated aorta may be managed by observation. Aortic dilatation in CHD patients may cause aortic annular enlargement that will predispose them to severe AR. In such conditions, concomitant aortic valvuloplasty or aortic root replacement is required. The Bentall operation (65) is root replacement with an aortic tube graft and a mechanical or biological valve. Coronary ostia are reimplanted into the aortic graft. The remodeling method (Yacoub) (66) and reimplantation method (David) (67) have been developed in order to preserve the aortic valve. The David operation (67) is a valve-preserving root replacement where the native aortic valve is re-implanted inside a tube graft. These procedures are well-known valve-sparing operations. On the other hand, in the Ross procedure (68), a pulmonary autograft is used to replace the aortic root and the aortic valve. The RV outflow tract and pulmonary artery are reconstructed with a homograft or other valve conduits. Superior hemodynamics, freedom from anticoagulation and longevity of pulmonary autografts have been demonstrated in many reports.

\section{Conclusions}

A subset of adult patients with CHD exhibit ongoing dilatation of the aortic root that may be related to the intrinsic properties of the aortic root or hemodynamic abnormality right-to-left shunting. This aortic dilatation can induce aortic aneurysm, rupture and aortic regurgitation, and provoke left ventricular hypertrophy, reduced coronary artery flow and possibly, left ventricular failure. However, medical prophylaxis to prevent further dilatation of the aorta and indications for aortic root surgery in CHD patients with a dilated aorta have yet to be established.

\section{Acknowledgements}

None.

\section{Footnote}

Conflicts of Interest: The author has no conflicts of interest to declare. 


\section{References}

1. Niwa K, Perloff JK, Bhuta SM, et al. Structural abnormalities of great arterial walls in congenital heart disease. Light and electron microscopic analyses. Circulation 2001;103:393-400.

2. Niwa K. Aortopathy in congenital heart disease in adults: aortic dilatation with decreased aortic elasticity that impacts negatively on left ventricular function. Korean Circ J 2013;43:215-20.

3. Roberts CS, Roberts WC. Dissection of the aorta associated with congenital malformation of the aortic valve. J Am Coll Cardiol 1991;17:712-6.

4. McKusick VA. Association of congenital bicuspid aortic valve and Erdheim's cystic medial necrosis. Lancet 1972;1:1026-7.

5. Halushka MK, Angelini A, Bartoloni G, et al. Consensus statement on surgical pathology of the aorta from the Society for Cardiovascular Pathology and the Association For European Cardio-vascular Pathology: II. Noninflammatory degenerative diseases - nomenclature and diagnostic criteria. Cardiovascular Pathology 2016;25:247-57.

6. Niwa K. Pathological background. In: Aortopathy, Niwa K, Kaemmerer H (eds). Tokyo: Springer, 2017:15-30.

7. John AS, McDonald-McGinn DM, Zackai EH, et al. Aortic root dilation in patients with 22 q11.2 deletion syndrome. Am J Med Genet A 2009;149A:939-42.

8. Niwa K, Siu SC, Webb GD, et al. Progressive aortic root dilatation in adults late after repair of tetralogy of Fallot. Circulation 2002;106:1374-8.

9. Niwa K. Tetralogy of Fallot and pulmonary atresia with ventricular septal defect. In: Niwa K, Kaemmerer H (eds). Aortopathy. Tokyo: Springer, 2017:277-90.

10. Tan JL, Davlouros PA, McCarthy KP, et al. Intrinsic histological abnormalities of aortic root and ascending aorta in tetralogy of Fallot: evidence of causative mechanism for aortic dilatation and aortopathy. Circulation 2005;112:961-8.

11. Bahnson HT, Spencer FC, Landtman B, et al. Surgical treatment and follow-up of 147 cases of tetralogy of Fallot treated by correction. J Thorac Cardiovasc Surg 1962;44:419-32.

12. Rieker RP, Berman MA, Stansel HC Jr. Postoperative studies in patients with tetralogy of Fallot. Ann Thorac Surg 1975;19:17-26.

13. Chong WY, Wong WH, Chiu CS, et al. Aortic root dilation and aortic elastic properties in children after repair of tetralogy of Fallot. Am J Cardiol 2006;97:905-9.

14. Niwa K. Aortic root dilatation in tetralogy of Fallot longterm after repair-histology of the aorta in tetralogy of Fallot: evidence of intrinsic aortopathy. Int J Cardiol 2005;103:117-9.

15. Kim WH, Seo JW, Kim SJ, et al. Aortic dissection late after repair of tetralogy of Fallot. Int J Cardiol 2005;101:515-6.

16. Rathi VK, Doyle M, Williams RB, et al. Massive aortic aneurysm and dissection in repaired tetralogy of Fallot; diagnosis by cardiovascular magnetic resonance imaging. Int J Cardiol 2005;101:169-70.

17. Wijesekera VA, Kiess MC, Grewal J, et al. Aortic dissection in a patient with a dilated aortic root following tetralogy of Fallot repair. Int J Cardiol 2014;174:833-4.

18. Konstantinov IE, Fricke TA, d'Udekem Y, et al. Aortic dissection and rupture in adolescents after tetralogy of Fallot repair. J Thorac Cardiovasc Surg 2010;140:e71-3.

19. Dearani JA, Burkhart HM, Stulak JM, et al. Management of the aortic root in adult patients with conotruncal anomalies. Semin Thorac Cardiovasc Surg Pediatr Card Surg Annu 2009:122-9.

20. Egan M, Phillips A, Cook SC. Aortic dissection in the adult Fontan procedure with aortic root enlargement. Pediatr Cardiol 2009;30:562-3.

21. Stolla M, Sweeney A, Alfieris GM, et al. Aortic aneurysm with a ruptured dissection in a 15 -year-old boy with hypoplastic left heart syndrome. J Thorac Cardiovasc Surg 2014;147:e35-6.

22. Cleuziou J, Mayr N, Schreiber C, et al. Giant aortic aneurysm 18 years after repair of double-outlet right ventricle with pulmonary stenosis. Ann Thorac Surg 2006;82:e31-2.

23. Nowitz A. Acute ascending aortic dissection 41 years after mustard procedure. J Cardiothorac Vasc Anesth 2013;27:735-9.

24. Sharma R, Cohen B, Peters P, et al. Aortic valve-sparing in aortic dissection with transposition of great arteries. Asian Cardiovasc Thorac Ann 2013;21:202-4.

25. Gutierrez PS, Binotto MA, Aiello VD, et al. Chest pain in an adult with truncus arteriosus communis. Am J Cardiol 2004;93:272-3.

26. Dodds GA 3rd, Warnes CA, Danielson GK. Aortic valve replacement after repair of pulmonary atresia and ventricular septal defect or tetralogy of Fallot. J Thorac Cardiovasc Surg 1997;113:736-41.

27. Mizuno A, Niwa K, Matsuo K, et al. Survey of reoperation indications in tetralogy of Fallot in Japan. Circ J 
2013;77:2942-7.

28. Hinton RB, Martin LJ, Rame-Gowda S, et al. Hypoplastic left heart syndrome links to chromosomes $10 \mathrm{q}$ and $6 \mathrm{q}$ and is genetically related to bicuspid aortic valve. J Am Coll Cardiol 2009;53:1065-71.

29. Hinton RB, Martin LJ, Tabangin ME, et al. Hypoplastic left heart syndrome is heritable. J Am Coll Cardiol 2007;50:1590-5.

30. Cohen MS, Marino BS, McElhinney DB, et al. Neo-aortic root dilation and valve regurgitation up to 21 years after staged reconstruction for hypoplastic left heart syndrome. J Am Coll Cardiol 2003;42:533-40.

31. Cardis BM, Fyfe DA, Mahle WT. Elastic properties of the reconstructed aorta in hypoplastic left heart syndrome. Ann Thorac Surg 2006;81:988-91.

32. Schlangen J, Fischer G, Petko C, et al. Arterial elastance and its impact on intrinsic right ventricular function in palliated hypoplastic left heart syndrome. Int J Cardiol 2013;168:5385-9.

33. Voges I, Jerosch-Herold M, Wegner P, et al. Frequent dilatation of the descending aorta in children with hypoplastic left heart syndrome relates to decreased aortic arch elasticity. J Am Heart Assoc 2015;4:e002107.

34. Lalezari S, Hazekamp MG, Bartelings MM, et al. Pulmonary artery remodeling in transposition of the great arteries: relevance for neoaortic root dilatation. J Thorac Cardiovasc Surg 2003;126:1053-60.

35. Takeuchi T, Imai Y, Takanashi Y, et al. Histological study of the great arteries in patients with arterial switch operation. Act Ped Cardiol Jpn 1996;12:506-12.

36. Angeli E, Raisky O, Bonnet D, et al. Late reoperations after neonatal arterial switch operation for transposition of the great arteries. Eur J Cardiothorac Surg 2008;34:32-6.

37. van der Bom T, van der Palen RL, Bouma BJ, et al. Persistent neo-aortic growth during adulthood in patients after an arterial switch operation. Heart 2014;100:1360-5.

38. Schwartz ML, Gauvreau K, del Nido P, et al. Longterm predictors of aortic root dilation and aortic regurgitation after arterial switch operation. Circulation 2004;110:II128-32.

39. Losay J, Touchot A, Capderou A, et al. Aortic valve regurgitation after arterial switch operation for transposition of the great arteries: incidence, risk factors, and outcome. J Am Coll Cardiol 2006;47:2057-62.

40. McMahon CJ, Ravekes WJ, Smith EO, et al. Risk factors for neo-aortic root enlargement and aortic regurgitation following arterial switch operation. Pediatr Cardiol 2004;25:329-35.
41. Ono M, Goerler H, Boethig D, et al. Valve-sparing operation for aortic root aneurysm late after Mustard procedure. Ann Thorac Surg 2007;83:2224-6.

42. Yurasek GK, Gauvreau K, Powell AJ, et al. Great vessel root and artery dimensions in transposition of the great arteries repaired with atrial switch operation. Pediatr Cardiol 2014;35:457-62.

43. Lambert E, d'Udekem Y, Cheung M, et al. Sympathetic and vascular dysfunction in adult patients with Fontan circulation. Int J Cardiol 2013;167:1333-8.

44. Tomkiewicz-Pajak L, Dziedzic-Oleksy H, Pajak J, et al. Arterial stiffness in adult patients after Fontan procedure. Cardiovasc Ultrasound 2014;12:15.

45. Müller J, Ewert P, Hager A. Increased aortic blood pressure augmentation in patients with congenital heart defects - A cross-sectional study in 1125 patients and 322 controls. Int J Cardiol 2015;184:225-9.

46. Bhat DP, Gupta P, Aggarwal S. Elevated aortic augmentation index in children following Fontan palliation: Evidence of stiffer arteries. Pediatr Cardiol 2015;36:1232-8.

47. Khairy P, Fernandes SM, Mayer JE Jr, et al. Long-term survival, modes of death, and predictors of mortality in patients with Fontan surgery. Circulation 2008;117:85-92.

48. Ohuchi H, Hayama Y, Negishi J, et al. Determinants of aortic size and stiffness and the impact on exercise physiology and renal function in patients after the Fontan procedure operation. Int Heart J 2017;58:73-80.

49. Jin SM, Noh CI, Bae EJ, et al. Impaired vascular function in patients with Fontan procedure circulation. Int J Cardiol 2007;120:221-6.

50. Carlo WF, McKenzie ED, Slesnick TC. Root dilation in patients with truncus arteriosus. Congenit Heart Dis 2011;6:228-33.

51. Henaine R, Azarnoush K, Belli E, et al. Fate of the truncal valve in truncus arteriosus. Ann Thorac Surg 2008;85:172-8.

52. Habashi JP, Judge DP, Holm TM, et al. Losartan, an AT1 antagonist, prevents aortic aneurysm in a mouse model of Marfan syndrome. Science 2006;312:117-21.

53. Brooke BS, Habashi JP, Judge DP, et al. Angiotensin II blockade and aortic-root dilation in Marfan syndrome. N Engl J Med 2008;358:2787-95.

54. Phomakay V, Huett WG, Gossett JM. $\beta$-Blockers and angiotensin converting enzyme inhibitors: comparison of effects on aortic growth in pediatric patients with Marfan syndrome. J Pediatr 2014;165:951-5.

55. Lacro RV, Dietz HC, Sleeper LA, et al. Atenolol versus 
losartan in children and young adults with Marfan's syndrome. N Engl J Med 2014;371:2061-71.

56. Nolte JE, Rutherford RB, Nawaz S, et al. Arterial dissections associated with pregnancy. J Vasc Surg 1995;21:515-20.

57. Manalo-Estrella P, Barker AE. Histologic findings in human aortic media associated with pregnancy. Arch Pathol 1967;83:336-41.

58. Katsuragi S, Ueda K, Yamanaka K, et al. Pregnancyassociated aortic dilatation or dissection in Japanese women with Marfan syndrome. Circ J 2011;75:2545-51.

59. Donnelly RT, Pinto NM, Kocolas I, et al. The immediate and long-term impact of pregnancy on aortic growth rate and mortality in women with Marfan syndrome. J Am Coll Cardiol 2012;60:224-9.

60. Shotan A, Widerhorn J, Hurst A, et al. Risks of angiotensin- converting enzyme inhibition during pregnancy: experimental and clinical evidence, potential mechanisms, and recommendations for use. Am J Med 1994;96:451-6.

61. Erbel R, Aboyans V, Boileau C, et al. 2014 ESC guidelines on the diagnosis and treatment of aortic disease. Eur Heart J 2014;35:2873-926.

62. Hiratzka LF, Bakris GL, Beckman JA, et al. 2010 ACCF/AHA/AATS/ACR/ASA/SCA/SCAI/SIR/STS/ SVM Guidelines for the diagnosis and management of patients with thoracic aortic disease. A Report of the

Cite this article as: Niwa K. Aortic dilatation in complex congenital heart disease. Cardiovasc Diagn Ther 2018;8(6):725-738. doi: $10.21037 /$ cdt.2018.12.05
American College of Cardiology Foundation/American Heart Association Task Force on Practice Guidelines, American Association for Thoracic Surgery, American College of Radiology, American Stroke Association, Society of Cardiovascular Anesthesiologists, Society for Cardiovascular Angiography and Interventions, Society of Interventional Radiology, Society of Thoracic Surgeons, and Society for Vascular Medicine. J Am Coll Cardiol 2010;55:e27-129.

63. Niwa K. Landmark lecture: Perloff lecture: Tribute to Professor Joseph Kayle Perloff and lessons learned from him: aortopathy in adults with CHD. Cardiol Young 2017;27:1959-65.

64. Stulak JM, Dearani JA, Burkhart HM, et al. Does the dilated ascending aorta in an adult with congenital heart disease require intervention? J Thorac Cardiovasc Surg 2010;140:S52-7.

65. Bentall H, De Bono A. A technique for complete replacement of the ascending aorta. Thorax 1968;23:338-9.

66. Sarsam MA, Yacoub M. Remodeling of the aortic valve anulus. J Thorac Cardiovasc Surg 1993;105:435-8.

67. David TE, Feindel CM, Bos J. Repair of the aortic valve in patients with aortic insufficiency and aortic root aneurysm. J Thorac Cardiovasc Surg 1995;109:345-51.

68. Ross DN. Replacement of aortic and mitral valves with a pulmonary autograft. Lancet 1967;2:956-8. 